\title{
Osteosarcoma of maxilla: A case report
}

\author{
Dr M.Vijaya Sree M.D ${ }^{1}$, Dr I .V.Renuka. M.D, ${ }^{2}$ Dr.G. Saila bala. M.D, ${ }^{3}$ \\ Dr K. Nageswara rao M.D, ${ }^{4}$ \\ ${ }^{I}$ Associate Professor of pathology, Guntur Medical college,Guntur,Andhra pradesh,India. \\ ${ }^{2}$ Professor of Pathology, Guntur Medical college, Guntur, Andhra pradesh,India \\ ${ }^{3}$ Professor of Pathology, Guntur Medical colPlege, Guntur, Andhra pradesh,India \\ ${ }^{4}$ Assistant Professor of pathology, Guntur Medical college, Guntur, Andhra pradesh,India
}

\begin{abstract}
Craniofacial Osteosarcoma is a relatively rare entity comprising abourt 6-10\% of all sarcomas. We present a rare case of maxillary OS early diagnosed in a 17 year old girl throught clinical, radiological and histological examination.The patient was much younger than the average age of presentation of a jaw tumor, which typically presents in the third to fourth decade of life.
\end{abstract}

\section{Introduction}

Osteosarcoma is one the most common primary malignant neoplasms, which predominantly occur in the long bones and rarely in the maxilla, facial area (6-10\% of all sarcomas $)^{(1)}$ With in the craniofacial region the mandible is usually reported as the most common site of the involvement,followed by the maxilla and skull. ${ }^{(1,2)}$ Jaw lesions are diagnosed on average two decade later than sarcomas of long bones, with a peak incidence between 20 and 40 years ${ }^{(3)}$. Head and neck OS (HNOS) are as associated with a lower metastatic rate than long bone OS, and they have a better 5year survival,ranging between $27 \%$ and $84 \%$.Approximately $80 \%$ of HNOS originate from soft tissue, while $20 \%$ arise from bone.Ahthough a male predilection has been reported,some authors have observed a slight predominance in females ${ }^{(4)}$.The cheif clinical features are swelling,pain and ulceration ${ }^{(5)}$.Radiologically, the findings may include radiolucency,radiopacity,or a mixture of both with poorly defined irregular margin. ${ }^{(5)} \mathrm{HNOS}$ differ from Osteosarcoma of the long bones in its biological behaviour, presenting a lower incidence of metastasis and a better prognosis.Early diagnosis and adequate surgical resection are the keys to high survival rates ${ }^{(6)}$

\section{Case report}

A 17 year old girl presented with swelling over the left side of cheek since three weeks. It started intraorally and gradually it increased in size and was exhibited extraorally.There was no discharge from the swelling.It was associated with difficulty in swallowing solids, There was no history of other medical problems and she was otherwise in a state of good health.The hematological and biochemical parameters were with in normal limits.All the vital signs like blood pressure,pulse,respiratory rate and temparature were with in normal limits.On inspection a diffuse swelling was noted on the left side cheek over the body of the maxilla measuring $4 \mathrm{~cm} \times 3 \mathrm{~cm} \times 2 \mathrm{~cm}$ in dimensions. The skin over the swelling was normal in appearance.The surface over the swelling showed no secondary changes like scar formation,sinus or fistula with no discharge of any kind. There was no localized rise in temparature. The swelling was firm, immovable and was fixed to the underlyimg bone. On internal examination a solitary localized swelling over upper left molar tooth extending in to maxilla and retromolar area.The surface of the swelling was red in color.On palpation swelling was firm in consistency,non tender and fixed to the underlying bone.All the thease features gave a clinical impression of a neoplastic swelling.To confirm the nature of the swelling various investigations were carried out.The patient was first subjected to radio graphic investigations.A CT scan of the paranasal sinus region showed a $3 \mathrm{~cm} \times 3 \mathrm{~cm}$ lesion in the body of the maxilla with bony erosion in to lateral wall.An incisional biopsy was carried out under anesthesia and sent to histopathological examination. We received multiple greywhite to gery brown bits of total size $2.5 \mathrm{~cm} \times 2 \mathrm{~cm} \times 1 \mathrm{~cm}$. The histological examination revealed areas of osteoid surrounded by sheets of neoplastic cells.Microscopic diagnosis was osteosarcoma.Immunohistochemistry,pancytokeratin was also done to differentiate it from malignant odentogenic tumor.The pancytokeratin was negative in neoplastic cells. Thus the final diagnosis of osteosarcoma was confirmed.The patient was referred to the oncology hospital for further treatment. 


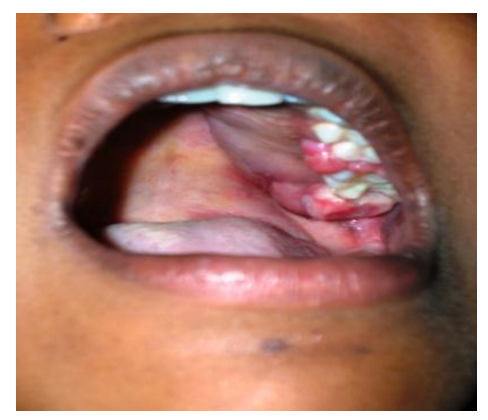

Figure 1: Intraoral view of the tumor

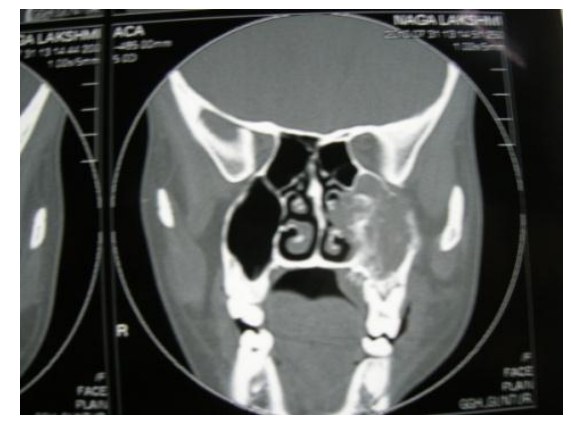

Figure 2: C T scan showing extensive maxillary tumor

\section{Discussion}

Os involves the head and neck region and approximately $10 \%$ of all sarcomas. The mandible and maxilla are the most frequently affected sites,followed by the paranasal sinuses and skull ${ }^{(6)}$. The HNOS occur in a population older than the one in which sarcoma of the long bones occur, with a peak incidence from $20-$ 40 years.Males are affected slightly more often than females.Approximately $80 \%$ of HNOS originate from soft tissue,while20\% arise from bone. ${ }^{(7)}$ soft tissue sarcomas arise from the mesenchyme,including muscle,endothelial cells,cartilage and supporting elements. ${ }^{(8)}$

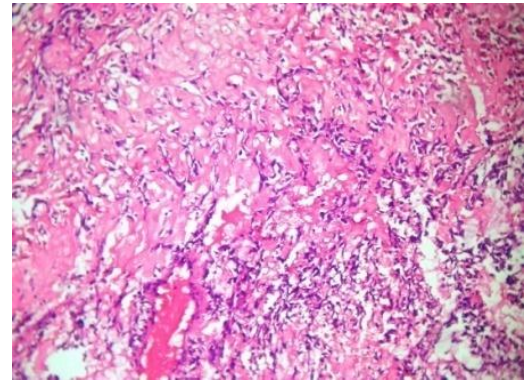

Figure 3:Hematoxalin and eosin stains Magnific ation 100x. Note areas of osteoid formation pleomorphism and hyperchromasia

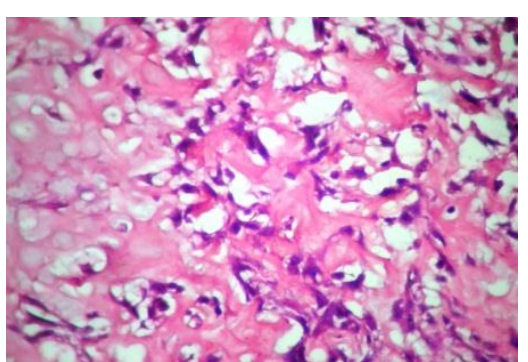

Figure4: Hematoxalin and eosin stains Magnific ation 400x. Note moderate nuclear

Most head and neck sarcomas appear at presentation as localized disease.Regional metastasis occur in 10-15\% of overall HNOS; most of the these arise from high grade primary lesions. At presentation,distant metastasis are rare in absence of regional metastasis; the presence of nodal metastasis showed a prompt search for distant metastasis. Most common site of distant metastasis is the lung, followed by liver and bone. The diagnosis of osteosarcoma is usually dependent on clinical,radiologic and histopathological features ${ }^{(9)}$ The affected patients usually present with pain and swelling of the concerned area,along with occasional ulveration. ${ }^{(10)}$ Other clinical features include mobility of tooth , numbness with the lips $\{$ or $\}$ other parasthesia. ${ }^{\{11\}}$ Radiological evaluation is necessary for diagnosis because clinical symptoms such as pain, parasthesia, swelling etc, are non specific ${ }^{\{12\}}$ Radiographically may present with an expansion of bones, it may present as radio opacity, radio lucency \{or\}of mixed nature.There is a need to be more consicous while diagnosing such patients as it often goes undiagnosed for a significant period of time. This is so because some of the signs and symptoms can be produced by a number of different developmental, infections, benign neoplastic diseases \{or\} malignancies. Further its presentation is similar to that of osteomyelitis, ossifying fibroma and fibrous dysplasia which has often causes an osteosarcoma to be delayed in its diagnosis. A tissue biopsy is the only means of making a definite diagnosis and such cases presenting clinically of ostreosarcoma requires a biopsy as soon as possible. ${ }^{\{13\}}$ The biopsy specimen from superfaecial \{or\} peripheral aspects of the tumour from the advancing edge is least likely to be representative of the tumour and frequently fails to demonestrate osteoid formation. The deeper biopsy should be taken from the centre of the lesion. This will avoid missing the diagnostic portion of the tumour because superfaecial part of the specimen include the immature part of the lesion. Early and small lung metastatic deposits are of great concern in osteosarcoma. So its complete screening requires a chest radiography \{or $\}$ a chest CT scan. ${ }^{\{13\}}$ The histopathological evaluation of the biopsy specimen showed a cellular stroma with evidence of osteoid and chondroid formation.The former is a diagnostic feature for the diagnosis of osteosarcoma.The tumor classification as osteoblastic (or) chondroblastic was not perfomed,since the specimen was collected by an incisional biopsy.More ever no association between histological classification JOS and 
survival has been reported.Regardless of favourable biologic behaviour in relation to other skeletal bone osteosarcomas patients with JOS may exhibit advanced tumors mainly early diagnosis is not perfomed.

\section{Conclusion}

It is concluded that misdiagnosis is very common in osteosarcoma of the jaw although JOS presents a better diagnosis than osteosarcoma of the long bones , patients with JOS can exhibit advanced tumors mainly early diagnosis is not performed. Clinicians and pathologists should be aware of its chrectorstics and main differential diagnosis to avoid late recognisition.IHC in our case prooved to be useful in confirmation of diagnosis. Although the histological picture of OS long bones and jaw bones exhibit similarity the management modalities may not necessarily the same. Since local regional recurrence is the main cause of mortality in OSJ,meticulous wide resection seem to be the main stay of treatment. Surgery with wide margins is an important factor is sucessful therapy for patient with JOS. Therefore early diagnosis and radical surgery with wide surgical margin are the key to a good outcome. In our view the presented case is interesting in terms of relatively early age of onset of tumour and diagnosis. CT has an important role in early diagnosis and the evaluation of its extent for the surgical planning.

\section{References}

[1]. Barnes EL,Peel RL,VerbinRS,Goodman MA, Apple BN,Diseases of the bones and joints. In: Barnes EL, editor , surgical pathology of the head and neck, NEWYORK: Marcel Dekker Inc; 1985.P.985-99.

[2]. MardingerO, Givol N,Talmi Yp, Taicher S, Saba K, Hashomer T.Osteosarcoma of jaw oral surg oral med oral pathol oral radiol Endod 2001;91;445-51.

[3]. Tommaso cuttilli, secondo scarsella, Desiderio Di Fabio,Antonio oliva ,pasqualino Cargini.Annals of maxillofacial surgery. JulyDecember 2011; volume1: Issue-2:176-80.

[4]. Bennett JH,Thomas G,Evans AW; SpeightPM. Osteosarcoma of the jaw ; a 30 year Retrospective review . Oral surg oral med oral pathol oral raidiol endod.2000 sep;90\{3\}:323-32.

[5]. August M, Magennis P, Decoitt D. Osteosarcoma of the jaws : Factors influencing prognosis. Int J oral maxillofac surg,1997 june;26\{3\}:198-204.

[6]. Kassir RR, Rassekh CH,kinsella JB,Segas J ,Carrau RL,Hookanson JA,Osteosarcoma of the head and neck: meta -analysis of non randomized studies.Laryngoscope 1997;107;56-61.

[7]. Yamaguchi s, nagasawa H,Suzuki,T,Fujji E,Iwaki H, Jakagi M,et al.Sarcomas of the oral and maxillofacial region; A review of 32 cases in 25 yrs. Clin oral investi of 2004;8:52-5.

[8]. Seidl RO, Gerein V, Vogel HJ, ernst A, mesenchymal chondrosarcoma of the facial skull. HNO 2001;49;744-9.

[9]. Bianchi SD, Boccardi A,. Radiological aspects of osteosarcoma of the jaws. Dento maxillofac Radiol 1999;28;42-7.

[10]. Amaral MB,Buchholz-J,Freire - maiaB,Reher P,desouza PE, Marigo Hde A,et al Advanced osteorsarcoma of the maxilla; a case report . med oral pathol ,oral cir Buccal ;2008;3E492-5.

[11]. Yesilova E, Akgunlu F, dolan maz D,Yasar F, sener S. Osteosarcoma; case report.EUR J dent 2007; 1:60-3.

[12]. Forteza G, colmenero B, lopez-barea F, Ostero sarcoma of maxialla and mandible . oral surg oral med oral pathol 1986;62;179-84.

[13]. Soheyl sheikh,shambulingappa pallagatti ,Amit Aggarwal, Deepak Gupta, Nidhi pari, Amit mittal . Osteosarcoma of maxilla. A case report $\mathrm{J}$ clin exp dent. 2012;2\{3\}; e117-20. 